\title{
Los géneros de Heliotropiaceae en Argentina
}

\author{
FEDERICO LUEBERT ${ }^{1}$
}

\begin{abstract}
Resumen: La delimitación genérica dentro de Heliotropiaceae ha sufrido cambios en el último tiempo que no son recogidos en publicaciones taxonómicas recientes sobre el grupo en Argentina. En este trabajo se actualiza la taxonomía de los géneros argentinos de Heliotropiaceae y se enumeran las especies aceptadas en cada uno de ellos. Se proporciona una breve descripción para cada género y una clave para diferenciarlos. Los géneros de Heliotropiaceae aceptados en este trabajo son Euploca, Heliotropium, Ixorhea y Myriopus, todos presentes en Argentina. Se designan lectotipos para Heliotropium hasslerianum (EEuploca hassleriana) y Heliotropium patagonicum y se propone la nueva combinación Heliotropium lilloi (basada en Tournefortia lilloi) para completar el cuadro taxonómico de la familia en Argentina.
\end{abstract}

Palabras clave: Boraginaceae, Boraginales, Heliotropium, Taxonomía, Tournefortia

Summary: The genera of Heliotropiaceae in Argentina. The generic delimitation within Heliotropiaceae has changed in the past years. However, these changes are not reflected in recent taxonomic publications on the group in Argentina. In this contribution, the taxonomy of the genera of Heliotropiaceae in Argentina is updated and all accepted species are enumerated. Brief generic descriptions and a key to distinguish the genera are provided. The genera of Heliotropiceae accepted in this work are Euploca, Heliotropium, Ixorhea and Myriopus, all of them present in Argentina. Lectotypes for Heliotropium hasslerianum (EEuploca hassleriana) and Heliotropium patagonicum are here selected and the new combination Heliotropium lilloi (based on Tournefortia lilloi) is proposed in order to complete the taxonomic treatment of the family in Argentina.

Key words: Boraginaceae, Boraginales, Heliotropium, Taxonomy, Tournefortia

\section{INTRODUCCIÓN}

En un artículo reciente publicado en esta revista (Di Fulvio \& Ariza Espinar, 2016) se revisan las especies argentinas del género Heliotropium L., trabajo que completa el tratamiento de Boraginaceae s.l. presentado anteriormente por estos autores (Ariza-Espinar, 2006). Dicho trabajo constituye un valioso aporte a la sistematización de la información taxonómica sobre este grupo en Argentina en la medida que se proporcionan descripciones, ilustraciones, sinonimia y datos de distribución para todas las especies mencionadas. En esos trabajos se sigue un

\footnotetext{
1 Universität Bonn, Nees-Institut für Biodiversität der Pflanzen, Meckenheimer Allee 170, D-53115 Bonn, Germany. Universidad de Chile, Departamento de Silvicultura y Conservación de la Naturaleza, Santiago, Chile.Email: fluebert@uni-bonn.de
}

concepto amplio y tradicional de Boraginaceae s.l. con cuatro subfamilias (Boraginoideae, Cordioideae, Ehretioideae y Heliotropioideae). Este concepto excluye Hydrophyllaceae y otros taxones que forman un grupo monofilético junto con Boraginaceae s.l. (Ferguson, 1999; Gottschling et al., 2001; Weigend et al., 2014; Refulio-Rodríguez \& Olmstead, 2014; Stull et al., 2015). Esta circunscripción amplia de Boraginaceae s.l. ha llevado a algunos autores a elevar las subfamilias antes mencionadas al nivel de familia y a tratar el orden Boraginales como compuesto de once familias (Luebert et al., 2016). El autor de este trabajo es partidario de tratar Boraginaceae subfam. Heliotropoideae al nivel de familia y los argumentos para ello han sido expuestos en la publicación mencionada arriba (Luebert et al., 2016), a la que el lector es referido. Sin embargo, la aceptación o rechazo de este punto de vista no afectan las consideraciones que a continuación se exponen. 
En lo relativo a la familia Heliotropiaceae, donde está el foco de la presente contribución, Ariza-Espinar (2006) y Di Fulvio \& Ariza Espinar (2016) siguen la propuesta presentada por Johnston (1928, 1930), donde la familia estaría compuesta por dos grandes géneros, Heliotropium y Tournefortia L., además de Ixorhea Fenzl, género monotípico y endémico de Argentina. La propuesta de Johnston $(1928,1930)$ es también seguida y complementada por Förther (1998), en cuya clasificación se reconocen ocho géneros en Heliotropiaceae: Argusia Böhm., Ceballosia Kunkel Heliotropium, Hilgeria Förther, Ixorhea, Nogalia Verdc., Schleidenia Endl. y Tournefortia. De ellos los tres géneros antes mencionados estarían presentes en Argentina, tal como está presentado por Ariza-Espinar (2006). Estudios filogenéticos (Diane et al., 2002; Hilger \& Diane, 2003; Luebert et al., 2011; Weigend et al., 2014) muestran claramente que es posible reconocer dos grandes clados en Heliotropiaceae: uno compuesto por las especies tradicionalmente adscritas a Tournefortia sect. Cyphocyema I.M.Johnst., Heliotropium sect. Orthostachys (R.Br.) G.Don y los géneros Hilgeria, Ixorhea y Schleidenia y el otro compuesto por las restantes secciones de Heliotropium, Tournefortia sect. Tournefortia y los géneros Argusia, Ceballosia y Nogalia. Basado en estos resultados, donde los dos géneros más diversos de la familia, Heliotropium y Tournefortia, son polifiléticos, Craven (2005) propuso reunir a la familia completa dentro de Heliotropium. Sin emabrgo, propuestas más recientes que toman en consideración la morfología y relaciones filogenéticas (Diane et al., 2016; Luebert et al., 2016) proponen reconocer cuatro géneros monofiléticos y morfológicamente coherentes: Euploca Nutt., Heliotropium, Ixorhea y Myriopus Small. El primero de ellos incluye las especies tradicionalmente adscritas a Heliotropium sect. Orthostachys, Hilgeria y Schleidenia. El segundo comprende el clado que incluye Heliotropium, Tournefortia sect. Tournefortia, Argusia, Ceballosia y Nogalia. El tercero es monotípico. El cuarto corresponde a Tournefortia sect. Cyphocyema. Los cuatro géneros están presentes en Argentina. Esta clasificación genérica de Heliotropiaceae está siendo aceptada por especialistas en los distintos grupos, en especial en relación con las circunscripciones genéricas de Euploca y Myriopus (véase Luebert \& Frohlich, 2016).

El propósito de este trabajo es presentar un cuadro sinóptico de los géneros de Heliotropiaceae presentes en Argentina de acuerdo con los aspectos arriba mencionados. Para cada género se proporciona una lista de los nombres de las especies aceptados de acuerdo con Ariza-Espinar (2006) y Di Fulvio \& Ariza Espinar (2016).

\section{Materiales y Métodos}

Este trabajo se basa en la revisión de la literatura taxonómica y sistemática sobre Heliotropiaceae en general (véase Förther, 1998 y Diane et al., 2016 y las referencias ahí citadas), y sobre las especies argentinas de la familia en particular, especialmente Johnston (1928), Johnston (1930), Gangui (1955), Ariza-Espinar (2006) y Di Fulvio \& Ariza Espinar (2016). Las descripciones de los géneros y la clave para su determinación se basan en Diane et al. (2016). Ejemplares tipo fueron revisados en los herbarios: A, B, BM, C, E, F, FI, G, GH, GOET, H, HAL, K, M, MA, P, S, SGO, US, W, WU.

\section{Resultados y Discusión}

En el siguiente tratamiento se enumeran los géneros y las especies de Heliotropiaceae en Argentina de acuerdo a los aspectos previamente mencionados. Para cada especie se indica el basiónimo, su tipo nomenclatural y nombre utilizado por Ariza-Espinar (2006) o Di Fulvio \& Ariza Espinar (2016), si es que este difiere del nombre empleado aquí, para que el lector pueda hacer uso de esos trabajos (claves, sinonimia, descripciones, ilustraciones, distribución) y enlazarlos con la clasificación que aquí se presenta.

Clave para los géneros de Heliotropiaceae en Argentina

1. Plantas glutinosas, olorosas. Limbo de la corola de más de $2 \mathrm{~cm}$ de diámetro. Frutos con 4 clusas aladas. Ixorhea

1'. Plantas no olorosas. Limbo de la corola de menos de $1.5 \mathrm{~cm}$ de diámetro. Frutos con clusas no aladas.

2

2. Anteras apicalmente glabras, con el conectivo no elongado y apicalmente libres. Embrión recto. ..

Heliotropium 


\section{F. Luebert - Los géneros de Heliotropiaceae en Argentina}

2'. Anteras apicalmente pubescentes o papilosas, con el conectivo elongado y apicalmente coherentes encerrando el tubo de la corola. Embrión curvo. ... 3

3. Hierbas o subarbustos no escandentes. Fruto seco. Euploca

3'. Lianas o arbustos subescandentes. Fruto carnoso.

Myriopus

Euploca Nutt., Trans. Amer. Phil. Soc. II, 5: 189. 1836. Especie tipo: Euploca convolvulacea Nutt.

Subarbustos o hierbas anuales o perennes, pubescentes o raramente glabros. Hojas alternas o raramente pseudo-opuestas, lineares a ovadas $\mathrm{u}$ obovadas. Inflorescencias no ramificadas o ramificadas 1-4 veces, ebracteadas o bracteadas, frondosas o reducidas a flores axilares solitarias. Cáliz lobado hasta $1 / 3$ a $1 / 2$ de su longitud. Corola blanca a amarilla, lóbulos suborbiculares a ovado-acuminados. Anteras con el conectivo largamente elongado en el ápice, apicalmente coherentes encerrando el tubo corolino. Frutos secos, dividiéndose en cuatro clusas uniseminadas, cada una con dos concavidades o máculas circulares en su cara abaxial, raramente drupas secas. Embrión curvo.

Género cosmopolita con cerca de 100 especies, once de ellas presentes en Argentina.

El género Euploca es frecuentemente utilizado en la literatura taxonómica actual (por ejemplo Melo \& Semir, 2010; Degen Naumann \& Melo, 2012; Feuillet, 2016). Di Fulvio \& Ariza Espinar (2016) proporcionan sinonimias detalladas para las especies, incluyendo algunos de los nombres actualmentes disponibles en Euploca para las especies consideradas por estos autores bajo Heliotropium sect. Orthostachys. La autoría correcta de Heliotropium sect. Orthostachys (aquí considerado como sinónimo de Euploca) es "(R. Br.) G.Don" y no "R.Br.", ya que Brown (1810: 493) denomina esta entidad sin ninguna asignación de rango taxonómico y solo Don (1838: 361) le asigna la categoría de sección.

Euploca aurata (Phil.) Frohlich, Darwiniana n.s. 4(2): 193. 2016.
Basiónimo: Heliotropium auratum Phil., Anales Mus. Nac., Santiago de Chile 1891: 56. 1891. Tipo: Argentina, Catamarca, Antofagasta de la Sierra, I-1885, F. Philippi s.n. (Lectotypus designado por Förther, 1998: 180, SGO 54425!; isolectotypi SGO 42267!, GH barcode GH00097639!, US 944535!, WU 0069886!).

Nombre utilizado por Di Fulvio \& Ariza-Espinar (2016): Heliotropium auratum Phil.

Euploca campestris (Griseb.) Diane \& Hilger, Bot. Jahrb. Syst. 125(1): 48. 2003.

Basiónimo: Heliotropium campestre Griseb., Abh. Königl. Ges. Wiss. Göttingen 19: 234. 1874. Tipo: Argentina, Cordoba in campis ab urbe meridionalibus, 1871, P.G. Lorentz 96 (Holotypus GOET barcode GOET004842!; isotypi B destruido [foto $\mathrm{F}$ neg. 17316!], CORD barcode CORD00006104 [foto!]).

Nombre utilizado por Di Fulvio \& Ariza-Espinar (2016): Heliotropium campestre Griseb.

Euploca catamarcense (I.M.Johnst.) Frohlich, Darwiniana n.s. 4(2): 193. 2016.

Basiónimo: Heliotropium catamarcense I.M.Johnst., Contr. Gray. Herb. 81: 59. 1928. Tipo: Argentina, Catamarca, Pilciao, 700 m, 20IV-1917, Jörgensen 1868 (Holotypus GH barcode GH00097604!; isotypi GH barcode GH00097605!, LIL barcode LIL001430 [foto!], LIL001431 [foto!], SI barcode SI001133 [foto!], SI001134 [foto!], SI001135 [foto!], US barcode US00110847!).

Nombre utilizado por Di Fulvio \& Ariza-Espinar (2016): Heliotropium catamarcense I.M.Johnst.

Euploca chrysantha (Phil.) Diane \& Hilger, Bot. Jahrb. Syst. 125(1): 48. 2003.

Basiónimo: Heliotropium chrysanthum Phil., Anales Univ. Chile 21: 401. 1862. Tipo: Argentina, Mendoza, 1860/61, W. Diaz s.n. (Holotypus SGO [foto + fragm. GH barcode GH00097608!], isotypus $\mathrm{B}$ destruido [foto $\mathrm{F}$ neg. 17317!], posibles isotypi $\mathrm{G}$ barcode G00177384!, P barcode P00610163!, W 0012896 !)

Nombre utilizado por Di Fulvio \& Ariza-Espinar (2016): Heliotropium chrysanthum Phil.

Euploca filiformis (Lehm.) J.I.M.Melo \& Semir, Kew Bull. 64(2): 288. 2009.

Basiónimo: Heliotropium filiforme Lehm., Gött. 
Gel. Anz. 3: 1515. 1817. Tipo: Venezuela, Orinoco, in arenosis, Humboldt 1202 (Lectotypus designado por Förther, 1998: 195, B-WILLD 3246-1!; isolectotypi MEL 133288 no visto, $\mathrm{P}$ barcode P00670717!; posible isolectotypus S 11-21347!)

Nombre utilizado por Di Fulvio \& ArizaEspinar (2016): Heliotropium filiforme Lehm.

Euploca hassleriana (Chodat) J.I.M.Melo \& Semir, Kew Bull. 64(2): 288. 2009.

Basiónimo: Heliotropium hasslerianum Chodat, Bull. Herb. Boissier 2. ser. 2: 817. 1902. Tipo: Paraguay, pr. Igatimi, IX-1898-1899, E. Hassler 4761 (Lectotypus aquí designado, G barcode G00095082!; isolectotypi B destruido [foto F neg 17324!], BAB barcode BAB00000730 [foto!], F-1547443 [foto!], G barcode G00095083!, G00095084!, G00095085!, GH barcode GH00097623 [foto!], K barcode K000583571 [foto!], MO-1834914 no visto, MPU barcode MPU019722 [foto!], NY barcode NY00335393 [foto!], P barcode P00610190 [foto!], P00610191 [foto!], S S-R-2840!, UC barcode UC944491 [foto!]).

Nombre utilizado por Di Fulvio \& ArizaEspinar (2016): Heliotropium hasslerianum Chodat

Comentario: El tipo de esta especie fue erróneamente indicado tanto por (Förther, 1998) como por Melo \& Semir (2009: 288). El tipo se encuentra en $\mathrm{G}$, donde se conservan varios sintipos correspondientes a la misma colecta (Hassler 4761). El lectotipo aquí designado corresponde al espécimen proveniente del herbario Hassler, visto por Chodat (véase Chodat \& Hassler, 1907).

Euploca mendocina (Phil.) Diane \& Hilger, Bot. Jahrb. Syst. 125(1): 48. 2003.

Basiónimo: Heliotropium mendocinum Phil., Anales Univ. Chile 21: 400. 1862. Tipo: Argentina, Mendoza, W. Diaz s.n. (Lectotypus designado por Förther, 1998: 206, SGO 54423!; posibles isolectotypi $\mathrm{P}$ barcode P00610201!, W 0049649!, WU 0069857!).

Nombre utilizado por Di Fulvio \& Ariza-Espinar (2016): Heliotropium mendocinum Phil.

Euploca ocellata (Cham.) J.I.M.Melo \& Semir, Kew Bull. 64(2): 289. 2009.

Basiónimo: Heliotropium ocellatum Cham.,
Linnaea 4: 463. 1829. Tipo: Basilia, Sellow s.n. [3579] (Lectotypus designado por Förther, 1998: 209, LE no visto; istolectotypi B destruido [foto F neg 17335!], BR barcode BR0000006967314 [foto!], E barcode E00394546 [foto!], G-DC barcode G00147693 [foto!], GH barcode GH00097724 [foto!], HAL barcode HAL0071612 [foto!], K barcode K000096805 [foto!], K000096806 [foto!], L barcode L0004006 [foto!], LE no visto, M barcode M0188051 [foto!], M0185296 [foto!], P barcode P00610211 [foto!], US barcode US00110868 [foto!], W 0049646 [foto!]).

Nombre utilizado por Di Fulvio \& Ariza-Espinar (2016): Heliotropium ocellatum Cham.

Euploca pallescens (I.M.Johnst.) J.I.M.Melo \& Semir, Kew Bull. 64(2): 289. 2009.

Basiónimo: Heliotropium pallescens I.M.Johnst., Contr. Gray Herb. 81: 65. 1928. Tipo: Paraguay, Gran Chaco, Loma Clavel, T. Rojas (Pl. Hassler. Parag. 2477) (Holotypus GH barcode GH00097727 [foto!]; isotypi $\mathrm{B}$ destruido [foto $\mathrm{F}$ neg 17336!], BM barcode BM000089627 [foto!], C barcode C10008745 [foto!], F 1547450 [foto!], G barcode G00095167, G00095168, G00095169, G00077106, G00077107, NY barcocde NY00039039 [foto!], P barcode P00610213 [foto!], S S04-2403!, US bracode US00110870 [foto!], W 1906-0000911 [foto!]).

Nombre utilizado por Di Fulvio \& Ariza-Espinar (2016): Heliotropium pallescens I.M.Johnst.

Euploca procumbens (Mill.) Diane \& Hilger, Bot. Jahrb. Syst. 125(1): 48. 2003.

Basiónimo: Heliotropium procumbens Mill., Gard. Dict., ed. 8. n. 10. 1768. Tipo: America australis [Colombia]: Carthagena, Houston s.n. (Holotypus BM barcode BM000953065!).

Nombre utilizado por Di Fulvio \& Ariza-Espinar (2016): Heliotropium procumbens Mill.

Euploca salicoides (Cham.) J.I.M.Melo \& Semir, Kew Bull. 64(2): 289. 2009.

Basiónimo: Heliotropium salicoides Cham., Linnaea 8: 117. 1833. Tipo: Brasília, Sellow, s.n. (Holotypus B destruido [foto F neg 17342!]; isotypi GH GH00097744 [foto!], US barcode US00110885 [foto!]).

Nombre utilizado por Di Fulvio \& Ariza-Espinar (2016): Heliotropium salicoides Cham. 


\section{F. Luebert - Los géneros de Heliotropiaceae en Argentina}

Heliotropium L., Sp. Pl. 1: 130. 1753. Especie tipo: Heliotropium europaeum L. (Lectotypus designado por Britton \& Brown, 1913: 73).

Árboles pequeños, arbustos, subarbustos, hierbas anuales o perennes o raramente lianas, pubescentes o raramente glabros. Hojas alternas o pseudoopuestas, raramente pseudo-ternadas, lineares a ampliamente ovadas u obovadas, membranáceas a coriáceas, raramente suculentas; margen entero u ondulado, raramente crenado, dentado o pinnatisecto, a veces revoluto. Inflorescencias generalmente ramificadas, ebracteadas, multifloras. Cáliz lobado hasta cerca de $1 / 2$ de su longitud. Corola generalmente blanca o verde, raramente amarilla, naranja o morada; lóbulos suborbiculares, oblongos a lanceolados. Anteras a veces con el conectivo elongado apicalmente y generalmente glabras o muy raramente pubescentes. Estilo largo, corto o invisible; estigma obtuso a largamente elongado. Frutos secos o carnosos; frutos secos separándose en dos clusas biseminadas o en cuatro clusas uniseminadas, raramente indivisos; frutos carnosos indivisos, blancos, usualmente compuestos de dos núculas biseminadas. Embrión recto.

Género cosmopolita con cerca de 300 especies, 21 de ellas presentes en Argentina

En la circunscripción actual de Heliotropium se incluye Tournefortia sect. Tournefortia (Luebert et al., 2011, 2016; Diane et al., 2016). La combinación de Tournefortia lilloi I.M.Johnst. bajo Heliotropium es por lo tanto necesaria para completar el cuadro taxonómico de las especies argentinas de Heliotropiaceae, pero su encuadre dentro de Heliotropium se deja pendiente hasta que la complejidad taxonómica y nomenclatural del grupo pueda ser integralmente abordada en una nueva clasificación infragenérica.

Dentro del género Heliotropium el trabajo de Di Fulvio \& Ariza Espinar (2016) señala que se siguió la clasificación infragenérica propuesta por Johnston (1928), lo cual se materializó de manera exacta. Sin embargo, hay algunos nombres de taxa infragenéricos utilizados por Johnston (1928) que Förther (1998) demostró que deben ser sinonimizados según en principio de prioridad, por existir nombres anteriores para los mismos taxones y en la misma categoría taxonómica. Ellos son Heliotropium sect. Heliophytum (Cham.) Griseb. (1862), para el que se debe utilizar Heliotropium sect. Heliotrophytum G.Don (1938) y Heliotropium sect. Halmyrophila I.M.Johnst. (1928), para el que se debe emplear Heliotropium sect. Platygyne Benth. (1868). Esta última sinonimia es mencionada por Di Fulvio \& Ariza Espinar (2016: 748), pero no fue incorporada en su tratamiento.

Heliotropium sect. Coeloma (DC.) I.M.Johnst., Contr. Gray. Herb. 81: 5. 1928. Especie tipo: Heliophytum monostachyum DC. (Lectotypus designado por Johnston, 1928: 5).

Dos especies incluidas por Di Fulvio \& Ariza Espinar (2016) como pertenecientes a la sección Coleoma (DC.) I.M.Johnst. deberían incluirse en la sección Platygyne (sub Halmyrophila). Ellas son Heliotropium patagonicum (Speg.) I.M.Johnst. y Heliotropium ruiz-lealii I.M.Johnst. Esto también es comentado por Di Fulvio \& Ariza Espinar (2016: 748), pero no implementado. En el caso de $H$. patagonicum estudios filogenéticos muestran claras afinidades con H. curassavicum L. (Hilger \& Diane, 2003; Luebert et al., 2011) y observaciones personales en material de herbario con frutos maduros (Weigend et al. 7012, BM, F, M, MSB, W) así como estudios carpológicos no publicados (Hartmut H. Hilger, comunicación personal) confirman dichas afinidades. En el caso de H. ruizlealii los antecedentes son más escasos, pero Förther (1998) ya había señalado su posición dentro de Platygyne tal como fue sugerido originalmente por Johnston (1959). Datos moleculares no publicados tomados de Di Fulvio \& O. Basso 1111 (MA) confirman las afinidades de $H$. ruiz-lealii con la sección Platygyne.

Heliotropium schreiteri I.M.Johnst., Contr. Gray Herb. 81: 10. 1928. Tipo: Argentina, Salta, Piguirendo, 500 m, 9-II-1925, R. Schreiter 3603 (Holotypus GH barcode GH00097745!; isotypi BAB barcode BAB00000731 [foto!], LIL barcode LIL001360 [foto!]).

Heliotropium transalpinum Vell., Fl. Flumin. 68. 1829. Tipo: Flora fluminensis, Icones vol. 2, tab. 40! (Lectotypus designado por Frohlich, 1981: 102). 
Heliotropium transalpinum var. arcanum $\mathrm{Di}$ Fulvio \& Ariza, Bol. Soc. Argent. Bot. 39(1-2): 119. 2004. Tipo: Argentina, Formosa, $3 \mathrm{~km}$ de San Ramón, en dirección a San Carlos por ruta a Misión Tacaaglé, 16-XI-1991, Fortunato et al. 2380 (Holotypus BAB barcode BAB00000061 [foto!]; isotypi $\mathrm{BAB}$ barcode $\mathrm{BAB} 00000062$ [foto!], SI no visto, MO-1192406 no visto, G no visto).

Heliotropium transalpinum var. tiaridioides (Cham.) I.M.Johnst., J. Arnold Arbor. 16: 186. 1935.

Basiónimo: Heliotropium tiaridioides Cham., Linnaea 4: 453. 1829. Tipo: Brasil, Sellow s.n. (Lectotypus designado por Förther, 1998: 224, LE no visto; isolectotypi $\mathrm{B}$ destruido [foto $\mathrm{F}$ neg 17348!], G [Herb. Boissier]!, G-DC barcode G00147350!, HAL barcode HAL0071594!, K barcode K000583583!, L barcode L0004024 [foto!], P barcode P00622825!, W 0033025!, posible isolectotypi E barcode E00781700!, K barcode K000583584!, K000583585!).

Nombre utilizado por Di Fulvio \& Ariza-Espinar (2016): Heliotropium tiaridioides Cham.

Heliotropium veronicifolium Griseb., Abh. Königl. Ges. Wiss. Göttingen 19: 232. 1874. Tipo: Argentina, Cordoba in campis prope urbem, 1871, P.G. Lorentz 91 (Holotypus GOET barcode GOET004845!; isotypi B destruido [foto F neg. 17350!], CORD barcode CORD00006101 [foto!]).

Heliotropium sect. Heliotrophytum G.Don, Gen. Hist. 4: 363. 1838. Especie tipo: Heliotropium sidaefolium Cham. (Lectotypus deignado por Förther, 1998: 81)

Heliotropium amplexicaule Vahl, Symb. Bot. 3:21, 1794. Tipo: Montevideo, P. Commerson s.n. (Holotypus C barcode C10008720!; isotypi MPU barcode MPU022410 [foto!], P barcode P00482088!, P-JU-6566!; posible isotypus G-DC barcode G00147911!).

Heliotropium leiocarpum Morong, Ann. New York Acad. Sci. 7: 168. 1893. Tipo: Paraguay, Asunción, 1-IV-1889, T. Morong 634 (Lectotypus designado por Johnston, 1928: 24, NY barcode NY00335396 [foto!]; isolectotypi BM barcode BM000089657!, E barcode E00781635!,
E00781636!, GH barcode GH00097641 [foto!], MSB barcode MSB002930!, NDG barcode NDG42357 [foto!], NY barcode NY00335394 [foto!], US barcode US00512996 [foto!]).

Heliotropium nicotianifolium Poir., Encycl. [J. Lamarck \& al.] Suppl. 3. 23. 1813. Tipo: Argentina, Buenos Ayres, Commerson s.n. (Holotypus P-JU-6568!; isotypi B destruido, FI-W barcode FI004997!, G-DC barcode G00147843!, MPU barcode MPU019715 [foto!], MPU019716 [foto!], $\mathrm{P}$ barcode P00482085!, P00482086!)

Heliotropium phylicoides Cham., Linnaea 4: 460. 1829. Tipo: Brasilia meridionalis, Sellow s.n. (Lectotypus designado por Förther, 1998: 212, LE no visto; isotypi B destruido [foto F neg. 17338!], G-DC barcode G00146886!, HAL barcode HAL0071614!, K [Herb. Hook. ex Herb. Cham.]!, $\mathrm{P}$ barcode P00482083!).

Heliotropium sect. Hypsogenia I.M.Johnst., Contr. Gray. Herb. 81: 12. 1928. Especie tipo: Heliotropium microstachyum Ruiz \& Pav.

Heliotropium abbreviatum Rusby, Mem. Torrey Bot. Club 4: 224. 1895. Tipo: Bolivia, Vic. Cochabamba, 1891, Bang 924 (Holotypus NY barcode NY00335383!; isotypi B destruido, BM barcode BM000992034!, E barcode E00781621!, F 162407!, G barcode G00169835!, GH barcode GH00096697!, K barcode K000583617!, LE no visto, MO-2516530 [foto!], MSB barcode MSB002989!, NY barcode NY00335384!, PH barcode PH00014183 [foto!], US 944668!, W 1892-9030!, WIS barcode 0255173WIS [foto!]).

Heliotropium microstachyum Ruiz \& Pav., Fl. Peruv. 2: 3, t. 110. 1799. Tipo: Tarma, I-1780 (Lectotypus designado por Luebert \& Hilger, 2014: 3, MA barcode MA814829!; isolectotypi B destruido [foto F neg. nr. 17333!], FI-W barcode FI 004998!, MA barcode MA814831!, MA814832!; posible isolectotypi B-WILLD 3237!, F 842502!, G [Herb. Boissier]!, M barcode M0188668!, MPU MPU019717 [foto!], P barcode P00610207!, S S-R-2846!, Dombey s.n., P barcode P00610206!, Dombey 366, G-DC barcode G00148000!, L L0004005 [foto!], M barcode M0188054!, P $\mathrm{P} 03877800$ !). 


\section{F. Luebert - Los géneros de Heliotropiaceae en Argentina}

Heliotropium sect. Plagiomeris I.M.Johnst., Contr. Gray Herb. 81: 16. 1928. Especie tipo: Heliotropium paronychioides A.DC.

Heliotropium geissei F.Phil., Anales Univ. Chile 90: 352. 1895. Tipo: Chile, Aconcagua, San Lorenzo, s.f., Geisse s.n. (Lectotypus designado por Johnston, 1928: 17 y refinado por Luebert \& Muñoz-Schick, 2014: 31-32, SGO 42247!).

Heliotropium kurtzii Gangui, Revista Fac. Ci. Exact. Fís. Nat. (Córdoba) 27: 520. 1955. Tipo: Argentina, Neuquén, Entre Norquín y Hualcupén, 19-22-III-1888, F. Kurtz 6286 (Holotypus CORD barcode CORD00003786, CORD00003787 [foto!]; isotypi G barcode G00169840!, SI barcode SI001136 [foto!], SI001137 [foto!], US barcode US00110859 [foto!]).

Heliotropium paronychioides A.DC., Prodr. 9: 565. 1845. Tipo: Chili, T. Bridges s.n. (Lectotypus designado por Förther, 1998: 211, G-DC barcode G00147493!; isolectotypus G barcode G00177391!).

Heliotropium pinnatisectum R.L.Pérez-Mor., Darwiniana 12: 629. 1963. Tipo: Argentina, Neuquén, Parque Nacional Laguna Blanca, en terreno arenoso a $200 \mathrm{~m}$ de la Laguna Blanca, 16XII-1961, R.L. Pérez-Moreau \& B. Piccinini 3322 (Holotypus SI barcode SI001138 [foto!]; isotypus $\mathrm{BAB}$ barcode BAB00000060 [foto!]).

Heliotropium sect. Platygyne Benth., Fl. Austral. 4: 392. 1868. Especie tipo: Heliotropium curassavicum L.

Heliotropium curassavicum L., Sp. Pl. 1:130. 1753. Tipo: Heliotropium curassavicum, foliis lini umbilicati, in Morrison, P1. Hist. 3: 452, sect. 11, t. 31, f. 12.1699 (Lectotypus designado por Verdcourt, 1991: 67; tipotypus OXF [Herb. Morrison] [foto!], fide Förther, 1998, Jarvis, 2007).

Heliotropium curassavicum var. argentinum I.M.Johnst., Contr. Gray Herb. 81: 15. 1928. Tipo: Argentina, Chaco, Las Palmas, X-1917, P. Jörgensen 2243 (Holotypus GH barcode GH00097612!; isotypus A barcode A00097613!)
Heliotropium curassavicum var. fruticulosum I.M.Johnst., Wrightia 2: 15. 1959. Tipo: Argentina, San Juan, Entre Balde y Tucunuco, 7-XII-1957, A. Ruiz-Leal \& F. Roig 18840 (Holotypus A barcode A00097614!).

Comentario: En el protólogo se indica que el holotipo se encuentra en LL. Sin embargo, de acuerdo a una etiqueta en el material de A por L. Woodruff \& T. Wendt de 2009 (ambos curadores de LL) se indica que este nunca llegó a ser depositado en LL y por lo tanto el material de A (donde Johnston trabajó, véase Correll, 1961) debe considerarse como el holotipo.

Heliotropium johnstonii Ragonese, Bol. Soc. Argent. Bot. 3: 80. 1950. Tipo: Argentina, Catamarca, entre Totoralejos y Casa de Piedra, 17-X-1947, Ragonese \& Piccinini 6031 (Holotypus BAB 69121 barcode BAB00000056-BAB00000059 [foto!]; isotypi CORD barcode CORD00003785 [foto!], GH GH00097631 [foto!]).

Comentario: El tipo de esta especie en BAB corresponde a cuatro ejemplares (BAB00000056BAB00000059) de la misma colecta y con el mismo número de herbario (BAB 69121). El material en GH tiene los mismos datos de fecha colector y número de colecta, pero una localidad diferente ("Entre El Tuscal y Mansilla"), que no es mencionada por Ragonese (1950).

Heliotropium patagonicum (Speg.) I.M.Johnst., Contr. Gray Herb. 81: 8. 1928.

Basiónimo: Valentina patagonica Speg., Anales Soc. Ci. Argent. 53: 78. 1902. Tipo: Argentina, Costa del Rio Chubut, XI-1897, [J. Valentín] s.n. (Lectotypus aquí designado, LP 006698 [foto!]).

Comentario: Para la designación del lectotipo se tomó en consideración la calidad del material y los datos de colectas, seleccionándose el ejemplar que, coincidiendo plenamente con lo indicado en el protólogo, es más completo.

Heliotropium ruiz-lealii I.M.Johnst., Wrightia 2: 13. 1959. Tipo: Argentina, San Juan, Entre Balde y Tucunuco, 7-XII-1957, A. Ruiz-Leal \& F. Roig 18837 (Holotypus A barcode A00097743 [foto!]; isotypus CORD barcocde CORD00003789 [foto!]).

Comentario: Aquí cabe lo mismo que para Heliotropium curassavicum var. fruticulosum citado arriba. 
Heliotropium sect. Tiaridium (Lehm.) Griseb., Fl. W. Ind. 485. 1862. Especie tipo: Heliotropium indicum L. (Lectotypus designado por Johnston, $1928: 18)$.

Heliotropium elongatum (Lehm.) I.M.Johnst., Contr. Gray Herb. 81: 19. 1928.

Basiónimo: Tiaridium elongatum Lehm., Pl. Asperif. Nucif. 16. 1818. Tipo: Brasil, locis arenosis, F.W. Sieber s.n. (Lectotypus designado por (Förther, 1998): 237, MEL 233325 no visto; isotypus B-WILLD 3228!)

Nombre utilizado por Di Fulvio \& Ariza-Espinar (2016): Heliotropium elongatum Hoffm. ex Roem. \& Schult. nom. inval. pro syn.

Heliotropium indicum L., Sp. Pl. 1: 130. 1753. Tipo: Habitat in India utraque (Lectotypus designado por Mill en Cafferty \& Jarvis, 2004: 802, BM Herb. Hermann 1: 9, No. 70 BM-000621256!)

\section{[Tournefortia sect. Tournefortia]}

Heliotropium lilloi (I.M. Johnst.) Luebert, comb. nov.

Basiónimo: Tournefortia lilloi I.M.Johnst., Contr. Gray. Herb. 92: 71. 1930. Tipo: Argentina, Tucumán, Tafí Viejo, 22-IX-1907, M. Lillo 7099 (Holotypus GH barcode GH00096930 [foto!]; isotypus CORD barcode CORD00003797 [foto!]).

Nombre utilizado por Ariza-Espinar (2006): Tournefortia lilloi I.M.Johnst.

Ixorhea Fenzl, Verh. Zool.-Bot. Ges. Wien 36: 287. 1886. Especie tipo: Ixorhea tschudiana Fenzl

Arbusto densamente glanduloso y cubierto de resina. Hojas alternas o pseudo-opuestas, lanceoladas, con margen entero. Inflorescencias ramificadas, ebracteadas, paucifloras. Cáliz tubular, lobado hasta $1 / 3$ a $1 / 4$ de su longitud. Corola blanquecina a rosa-violeta, con lóbulos triangulares y estivación conduplicado-torcida. Anteras con el conectivo largamente elongado en el ápice; conectivo no sobrepasando el ápice del estigma. Estilo largo; estigma elongado. Fruto seco que se divide en cuatro clusas uniseminadas y apicalmente aladas. Embrión recto.

Género monotípico y endémico de Argentina.
Ixorhea tschudiana Fenzl, Verh. Zool.-Bot. Ges. Wien 36: 288. 1886. Tipo: Argentina, [...] zwischen Palopintado \& El Carmen [...] Felsen. Tschudi s.n. (Holotypus W 0050064!).

Myriopus Small, Man. S.E. Fl. 1131. 1933. Especie tipo: Myriopus poliochros (Spreng.) Small (Lectotypus designado por Förther, 1998: 128).

Lianas, raramente arbustos subescandentes, glabros a seríceos. Hojas alternas a pseudoopuestas, lanceoladas a ovadas. Inflorescencias ramificadas, ebracteadas. Cáliz lobado hasta $1 / 2$ de su longitud o hasta la base. Corola naranja, amarilla o verdosa; lóbulos subulados con margen involuto; base del tubo corolino ensanchada. Anteras con el conectivo largamente elongado apicalmente, con ápice papiloso, apicalmente coherentes encerrando el tubo corolino. Estilo largo; estigma cilíndrico, truncado en el ápice. Frutos carnosos tetralobulados, blancos o anaranjados, compuesto de clusas uniseminadas. Embrión curvo.

Alrededor de 25 especies neotropicales, cuatro de ellas presentes en Argentina.

Myriopus breviflorus (DC.) Luebert, Darwiniana n.s. 4(2): 193. 2016.

Basiónimo: Tournefortia breviflora DC., Prodr. 9: 520. 1845. Tipo: Brazil, Rio [de Janeiro], 1834, Lund s.n. (Holotypus G-DC! [foto F neg 7760!, foto MSB!]; isotypus P barcode P03525470!).

Nombre utilizado por Ariza-Espinar (2006): Tournefortia breviflora DC.

Myriopus paniculatus (Cham.) Feuillet, J. Bot. Res. Inst. Texas 2(1): 264. 2008.

Basiónimo: Tournefortia paniculata Cham., Linnaea 4(4): 468. 1829. Tipo: Brasilia intertropica, Sellow s.n. (Holotypus B destruido [foto F neg. 1053!]; isotypus G barcode G00236172!)

Nombre utilizado por Ariza-Espinar (2006): Tournefortia paniculata Cham.

Myriopus paniculatus var. austrinus (I.M.Johnst.) Luebert, Darwiniana n.s. 4(2): 194. 2016.

Basiónimo: Tournefortia paniculata var. austrina I.M.Johnst., Contr. Gray. Herb. 92: 82. 1930. Tipo: Argentina, Tucumán, La Ramada, XII-1914, M. 


\section{F. Luebert - Los géneros de Heliotropiaceae en Argentina}

Lillo 3032M (Holotypus GH barcode GH00096176 [foto!]).

Nombre utilizado por Ariza-Espinar (2006): Tournefortia paniculata var. austrina I.M.Johnst.

Myriopus rubicundus (Salzm. ex DC.) Luebert, Taxon 60(3): 677. 2011.

Basiónimo: Tournefortia rubicunda Salzm. ex DC., Prodr. 9: 526. 1845. Tipo: Brasil, Bahia, Salzmann 372 (Lectotypus designado por Johnston, 1930: 85, G-DC barcode G00147148!; isolectotypi E barcode E00766000!, FI-W barcode FI005004!, H barcode H1535648!, HAL barcode HAL0113290!, HAL0098678!, K barcode K000583443!, K000583444!, MPU barcode MPU019536 [foto!], MPU019535 [foto!], P barcode P04037974!).

Nombre utilizado por Ariza-Espinar (2006): Tournefortia rubicunda Salzm. ex DC.

Myriopus salzmannii (DC.) Diane \& Hilger, Bot. Jahrb. Syst. 125(1): 47. 2003.

Basiónimo: Tournefortia salzmannii DC., Prodr. 9: 524. 1845. Tipo: Brasil, Bahia, 1830, Salzmann 370 (Lectotypus designado por Johnston, 1930: 87, G-DC barcode G00147194!; isolectotypi FI-W barcode FI005010!, H barcode H1535649!, HAL barcode HAL0098679!, HAL0113291!, K barcode K000583452!, M barcode M0187939 [foto!], MPU barcode MPU019537 [foto!], MPU019538 [foto!], P barcode P00836353!, W barcode W18890284059!)

Nombre utilizado por Ariza-Espinar (2006): Tournefortia salzmannii DC.

\section{Agradecimientos}

Agradezco a los curadores de los herbarios A, B, BM, C, E, F, FI, G, GH, GOET, H, HAL, K, M, MA, OXF, P, S, SGO, US, W, WU por sus facilidades para consultar el material ahí depositado. Agradezco también a Wilhelm Barthlott, Maximilian Weigend y Jun Wen por su apoyo en diferentes fases del desarrollo de este trabajo y a Stephen Harris y Serena Marner (OXF) por facilitar la imagen del tipótipo de Heliotropium curassavicum. Este trabajo recibió apoyo del projecto Biodiversität im Wandel der Akademie der Wissenschaften und der Literatur, Mainz y del programa SYNTHESYS http://www.synthesys.info/ que es financiado por la European Community Research Infrastructure
Action bajo los programas FP6 "Structuring the European Research Area" (grant GB-TAF-4514) y FP7 "Capacities" (grants ES-TAF-136, ACTAF-2001, FR-TAF-1977, SE-TAF-4797, GBTAF-4761).

\section{Bibliografía}

ARIZA-ESPINAR, L. 2006. 252. Boraginaceae, parte 1 (excepto Heliotropium). Pp. 3-55 in Ariza-Espinar, L., Calviño, A., di Fulvio, T. E. \& Dottori, N. (eds.). Flora Fanerogámica Argentina, Fasc. 97. Proflora, CONICET, Córdoba.

BRITTON, N. L. \& A. BROWN. 1913. An illustrated flora of the northern United States, Canada and the British possessions (2nd edition). C. Scribner's sons, New York.

BROWN, R. 1810. Prodromus florae Novae Hollandiae et insulae Van-Diemen. J. Johnson \& Co., London.

CAFFERTY, S. \& C. E. JARVIS. 2004. Typification of Linnaean plant names in Boraginaceae. Taxon 53: 799-805.

CHODAT, R. \& E. HASSLER. 1907. Plantae Hasslerianae. Impr. Romet, Genève.

CORRELL, D. S. 1961. Ivan Murray Johnston (18981960). Taxon 10: 1-8.

CRAVEN, L. A. 2005. Malesian and Australian Tournefortia transferred to Heliotropium and notes on delimitation of Boraginaceae. Blumea 50: 375381.

DEGEN NAUMANN, R. \& J. I. M. MELO. 2012. Nuevas combinaciones en el género Euploca Nutt. (Boraginaceae) del Paraguay. Candollea 67: 368.

DI FULVIO, T. E. \& L. ARIZA ESPINAR. 2016. Las especies argentinas de Heliotropium (Boraginaceae). Bol. Soc. Argent. Bot. 51: 745-787.

DIANE, N., H. FÖRTHER \& H. H. HILGER. 2002. A systematic analysis of Heliotropium, Tournefortia, and allied taxa of the Heliotropiaceae (Boraginales) based on ITS1 sequences and morphological data. Amer. J. Bot. 89: 287-295.

DIANE, N., H. H. HILGER, H. FÖRTHER, M. WEIGEND \& F. LUEBERT. 2016. Heliotropiaceae. Pp. 203-211 in Kadereit, J. W. \& Bittrich, V. (eds.). The families and genera of vascular plants, vol. 14. Springer International Publishing, Cham.

DON, G. 1838. A general history of the dichlamydeous plants. J.G. and F. Rivington, London.

FERGUSON, D. M. 1999. Phylogenetic analysis and relationships in Hydrophyllaceae based on ndhF sequence data. Syst. Bot. 23: 253-268.

FEUILLET, C. 2016. Two new combinations in Euploca Nutt. (Heliotropiaceae, Boraginales) and a conspectus of the species of the Guiana Shield area. 
PhytoKeys 61: 101-124.

FÖRTHER, H. 1998. Die infragenerische Gliederung der Gattung Heliotropium L. und ihre Stellung innerhalb der Subfam. Heliotropioideae (Schrad.) Arn. (Boraginaceae). Sendtnera 5: 35-241.

FROHLICH, M. W. 1981. Heliotropium. Pp. 70-104 in Gómez-Pompa, A. (ed.). Flora de Veracruz. Instituto Nacional de Investigaciones sobre Recursos Bióticos, Xalapa.

GANGUI, N. 1955. Las especies silvestres de Heliotropium de la República Argentina. Revista Fac. Cienc. Exac. Fís. Nat.(Córdoba) 17: 481-560.

GOTTSCHLING, M., H. H. HILGER, M. WOLF \& N. DIANE. 2001. Secondary structure of the ITS1 transcript and its application in a reconstruction of the phylogeny of Boraginales. Plant Biol. 3: 629636.

HILGER, H. H. \& N. DIANE. 2003. A systematic analysis of Heliotropiaceae (Boraginales) based on trnL and ITS1 sequence data. Bot. Jahrb. Syst. 125: 19-51.

JARVIS, C. 2007. Order out of chaos. Linnaean plant names and their types. The Linnean Society of London and Natural History Museum, London.

JOHNSTON, I. M. 1928. Studies in the Boraginaceae VII.1. The South American species of Heliotropium. Contrib. Gray Herb. 81: 3-73.

JOHNSTON, I. M. 1930. Studies in the Boraginaceae VIII. I. Observations on the species of Cordia and Tournefortia known from Brazil. 3. Treatment of Tournefortia. Contrib. Gray Herb. 92: 66-89.

JOHNSTON, I. M. 1959. Some noteworthy American Borages. Wrightia 2: 13-22.

LUEBERT, F., G. BROKAMP, J. WEN, M. WEIGEND \& H. H. HILGER. 2011. Phylogenetic relationships and morphological diversity in Neotropical Heliotropium (Heliotropiaceae). Taxon 60: 663-680.

LUEBERT, F., L. CECCHI, M. W. FROHLICH, M. GOTTSCHLING, C. M. GUILLiAMS, H. H. HILGER, K. E. HASENSTAB-LEHMAN, J. S. MILLER, M. MITTELBACH, M. NAZAIRE, M. NEPI, D. NOCENTINI, D. OBER, R. G. OLMSTEAD, F. SELVI, M. G. SIMPSON, K. SUTORÝ, B. VALDÉS, G. K. WALDEN \& M. WEIGEND [BORAGINALES WORKING GROUP]. 2016. Familial classification of the Boraginales. Taxon 65: 502-522.
LUEBERT, F. \& M. W. FROHLICH. 2016. Four new combinations in Argentinian Heliotropiaceae. Darwiniana n.s. 4: 192-194.

LUEBERT, F. \& H. H. HILGER. 2014. Typification of Heliotropium and Tournefortia (Heliotropiaceae) species described by Ruiz and Pavón. Anales Jard. Bot. Madrid 71: e012.

LUEBERT, F. \& M. MUÑOZ-SCHICK. 2014. Heliotropium geissei (Heliotropiaceae): nuevos registros, tipificación, posición sistemática y estado de conservación. Gayana Bot. 71: 30-36.

MELO, J. I. M. \& J. SEMIR. 2009. Two new Brazilian species and new combinations in Euploca (Heliotropiaceae). Kew Bull. 64: 285-289.

MELO, J. I. M. \& J. SEMIR. 2010. Taxonomia do gênero Euploca Nutt. (Heliotropiaceae) no Brasil. Acta Bot. Bras. 24: 111-132.

RAGONESE, A. E. 1950. Una nueva especie de Heliotropium de la flora argentina. Bol. Soc. Argent. Bot. 3: 80-82.

REFULIO-RODRÍGUEZ, N. F. \& R. G. OLMSTEAD. 2014. Phylogeny of Lamiidae. Amer. J. Bot. 101: 287-299.

STULL, G. W., R. DUNO DE STEFANO, D. E. SOLTIS \& P. S. SOLTIS. 2015. Resolving basal lamiid phylogeny and the circumscription of Icacinaceae with a plastome-scale data set. Amer. J. Bot. 102: 1794-1813.

VERDCOURT, B. 1991. Boraginaceae. Pp. 1-124 in Polhill, R. M. (ed.). Flora of Tropical East Africa. Royal Botanic Gardens, Kew.

WEIGEND, M., F. LUEBERT, M. GOTTSCHLING, T. L. P. COUVREUR, H. H. HILGER \& J. S. MILLER. 2014. From capsules to nutlets - Phylogenetic relationships in the Boraginales. Cladistics 30: 508518 .

Recibido el 2 de marzo de 2017, aceptado el 4 de mayo de 2017. 